\title{
MicroRNA-29b-3p enhances radiosensitivity through modulating WISP1-mediated mitochondrial apoptosis in prostate cancer cells
}

Aihong Mao ${ }^{1,2 \#, ~ J i n z h o u ~ T a n g 1 \#, ~ D e p i n g ~ T a n g ³, ~ F a n g ~ W a n g ², ~ S h i q i ~ L i a o ', ~ H o n g x i a ~ Y u a n ¹, ~ C a i p i n g ~ T i a n ¹, ~}$ Chao Sun ${ }^{2}$, Jing $\mathrm{Si}^{2}$, Hong Zhang ${ }^{2}$ and Xiaojun $\mathrm{Xia}^{1,4}$

1. Gansu Provincial Academic Institute for Medical Research, Lanzhou, China

2. Institute of Modern Physics, Chinese Academy of Sciences, Lanzhou, China.

3. School of Chemical \& Biological Engineering, Lanzhou Jiaotong University, Lanzhou 730070, PR China.

4. Gansu Provincial Cancer Hospital, Lanzhou, China.

\#These authors contributed equally to this work.

$\triangle$ Corresponding authors: Dr. Hong Zhang, Department of Heavy Ion Radiation Medicine, Institute of Modern Physics, Chinese Academy of Sciences, Lanzhou, 730000, China. E-mail: zhangh@impcas.ac.cn; Phone: +86 (931) 496-9344; Fax: +86 (931) 496-9170; Prof. Xiaojun Xia, Gansu Provincial Academic Institute for Medical Research, Gansu Provincial cancer hospital, 2 Xiaoxihu East Street, Lanzhou 730050, P.R. China. E-mail: xiaxiaojun2006@163.com; Phone: +86 (931) 230-2647; Fax: +86 (931) 230-2647.

(c) The author(s). This is an open access article distributed under the terms of the Creative Commons Attribution License (https://creativecommons.org/licenses/by/4.0/). See http:/ /ivyspring.com/terms for full terms and conditions.

Received: 2020.05.15; Accepted: 2020.08.26; Published: 2020.09.12

\begin{abstract}
Radiotherapy is frequently applied for clinically localized prostate cancer while its efficacy could be significantly hindered by radioresistance. MicroRNAs (miRNAs) are important regulators in mediating cellular responses to ionizing radiation (IR), and strongly associate with radiosensitivity in many cancers. In this study, enhancement of radiosensitivity by miR-29b-3p was demonstrated in prostate cancer cell line $\mathrm{LNCaP}$ in vitro. Results showed that miR-29b-3p expression was significantly upregulated in response to IR from both X-rays and carbon ion irradiations. Knockdown of miR-29b-3p resulted in radioresistance while overexpression of miR-29b-3p led to increased radiosensitivity (showing reduced cell viability, suppressed cell proliferation and decreased colony formation). In addition, miR-29b-3p was found to directly target Wntl-inducible-signaling protein 1 (WISPI). Inhibition of WISPI facilitated the mitochondrial apoptosis pathway through suppressing $\mathrm{Bcl}-\mathrm{XL}$ expression while activating caspase- 3 and poly (ADP-ribose) polymerase (PARP). The results indicated that miR-29b-3p was a radiosensitizing miRNAs and could enhance radiosensitivity of LNCaP cells by targeting WISPI. These findings suggested a novel treatment to overcome radioresistance in prostate cancer patients, especially those with higher levels of the WISPI expression.
\end{abstract}

Key words: MiR-29b-3p; WISP1; radiosensitivity; apoptosis; prostate cancer

\section{Introduction}

Prostate cancer is a prevalent cancer among men. In the United States, the number of new cases was $\sim 191,930$ whereas deaths account for 33,330 in 2020, which is showing an increasing incidence over the past number of years [1]. Radiotherapy is a frequently applied therapeutic intervention for localized prostate cancer. However, most patients typically develop radioresistance following a period of radiotherapy, leading to reduced efficacy [2]. Therefore, identification of novel approaches or agents to reverse the radioresistance of prostate cancer cells and improve the efficacy of radiotherapy is urgently required.

WISP1 (Wnt-1 inducible signaling pathway protein-1) belongs to the CCN family, which is regulated by the Wnt- $1 / \beta$-catenin pathway [3]. Overexpression of WISP1 not only could potentiate transformation and tumorigenesis, but also closely associated with poor prognosis in colon cancer, glioblastoma and oral squamous cell carcinoma [4-6]. 
A number of studies have demonstrated that WISP1 could facilitate prostate cancer cell growth and bone metastasis [7-9]. In addition, studies on esophageal carcinoma suggested that WISP1 was involved in mediating radioresistance [10-12], whilst the Wnt-1/ $\beta$-catenin pathway, which lies upstream of WISP1, was demonstrated to mediate radioresistance in glioblastoma [13] and breast cancer [14]. Therefore, targeting WISP1 may be a potentially useful approach for overcoming the radioresistance of prostate cancer cells.

Over the last decade, accumulating evidence has showed that microRNAs (miRNAs) serve pivotal roles in tumorigenesis and progression [15]. The dysregulation of miRNAs has been detected in many cancers [16]. Recently, we found several miRNAs, such as miR-449a and miR-16-5p, dysregulated in prostate cancer tissue or cells, where they served critical roles in response to ionizing radiation (IR) and associated strongly with radiosensitivity in prostate cancer cells [17-19]. These findings suggested that miRNAs have potential as novel targets for prostate cancer radiotherapy.

MicroRNA-29b (miR-29b) is a member of the miR-29 family (miR-29a, b, and c), which locates on two distinct chromosomes 1q32 and 7q32 [20]. Aberrant expression profiles of miR-29b has been found in many cancers, where the role of miR-29b in the development and progression of many types of malignancies has been extensively studied [21]. In prostate cancer, reduced expression of miR-29b or deletion was observed in tumor tissues or/and cell lines $[22,23]$. The expression of miR-29b was found to associate strongly with etiology, classification, progression, and prognosis of patients with prostate cancer [23]. Emerging evidences demonstrate that miR-29b inhibits prostate cancer cell proliferation and invasion by regulating different targets, including MCL-1, MMP-2 [24,25], DNMT3B and AKT3 [26]. MiR-29b was identified as a regulator of epithelialmesenchymal transition (EMT), which was involved in prostate cancers metastasis [27] and chemoresistance $[26,28]$. Although the function of miR-29b as a tumor suppressor was reported on multiple occasions to critically influence cancer progression and therapeutic outcome, it is still unclear whether WISP1 and miR-29b interact in response to IR. And whether miR-29b regulates radiosensitivity by targeting WISP1 in prostate cancer cells also remains to be fully elucidated.

Therefore, in this study, we investigated the expression and function of miR-29b-3p in response to IR in prostate cancer cells. Our results demonstrated that miR-29b-3p enhances IR-induced cell apoptosis and sensitizes LNCaP cells to $\mathrm{X}$-rays irradiation by targeting WISP1. The results highlighted a previously unrecognized mechanism involving miR-29b-3p and WISP1 in response to IR, and suggested a novel treatment option for prostate cancer patients, especially for those with higher levels of the WISP1 expression.

\section{Materials and Methods}

\section{Cell culture and irradiation treatment}

Human prostate cancer cell line LNCaP was obtained from the Cell Bank of Type Culture Collection of the Chinese Academy of Sciences. Cells were cultured in RPMI-1640 medium with 10\% FBS (Hyclone; GE Healthcare Life Sciences) and kept in a humidified $5 \% \mathrm{CO}_{2}$ incubator at $37^{\circ} \mathrm{C}$.

$\mathrm{X}$-rays were generated by an $\mathrm{X}$-ray machine (Faxitron RX-650) with $100 \mathrm{kVp}$, at a dose rate of 0.835 Gy/min. Carbon ion irradiation was performed on the Heavy Ion Research Facility in Lanzhou (HIRFL; Institute of Modern Physics, Chinese Academy of Sciences, Lanzhou, China) with energy $80.55 \mathrm{MeV} / \mathrm{u}$ and linear energy transfer was $59.75 \mathrm{keV} / \mu \mathrm{m}$, and dose rate was $2 \mathrm{~Gy} / \mathrm{min}$.

\section{RNA isolation and miRNA array analysis}

LNCaP cells with $80 \%$ confluence were seeded into $60-\mathrm{mm}$ dishes and stabilized overnight at $37^{\circ} \mathrm{C}$ and $5 \% \mathrm{CO}_{2}$. The cells were exposed to X-rays or carbon ion irradiation at a dose of 0.5 or $4 \mathrm{~Gy}$ at room temperature. $24 \mathrm{~h}$ after irradiation, total RNA samples were isolated using TRIzol ${ }^{\circledR}$ reagent (Invitrogen, Thermo Fisher Scientific, Inc.) and sent to Shanghai GeneChem Co., Ltd. for miRNA OneArray analysis, as previously described [19].

\section{Reverse transcription-quantitative PCR (RT-qPCR)}

Total RNA was isolated using Trizol reagent and reverse transcription reaction was performed using Mir- $X^{\mathrm{TM}}$ miRNA First Strand Synthesis Kit (Takara Bio., Inc.) according to manufacturer's protocol. RealTime PCR was carried out to measure the expression of miR-29b-3p using Mir- $X^{\mathrm{TM}}$ miRNA qRT-PCR Kit (Takara Bio., Inc.) with a specific sense primer (Table S1). WISP1 mRNA expression analysis was performed using Prime Script ${ }^{\mathrm{TM}}$ RT reagent Kit and gDNA Eraser and SYBR Advantage qPCR Premix (Takara Bio., Inc.) with specific primers (Table S2). The amplification reactions were performed using the QuantStudio ${ }^{\mathrm{TM}} 5$ Real-Time PCR System (Thermo Fisher Scientific, Inc.). The relative expression levels of miRNA and mRNA were evaluated using the $2^{-\Delta \Delta \mathrm{Ct}}$ method. RNU6 and GAPDH were used as references, respectively. 


\section{miRNA mimics and inhibitors transfection}

LNCaP cells with $30-40 \%$ confluence were incubated with antibiotic free RPMI-1640 medium for $24 \mathrm{~h}$ at $37^{\circ} \mathrm{C}$. Transfection was performed using the Trans IT®-2020 transfection reagent (Mirus Bio LLC) according to manufacturer's protocol. The sequences of the miR-29b-3p mimics (miR-29b-3p), inhibitors (anti-miR-29b-3p) and corresponding negative control (miR-con or anti-con) (RIBO Bio., Guangzhou) were presented in Table S3. MiR-29b-3p mimics and inhibitors were transfected in $\mathrm{LNCaP}$ cells at a final concentration of 50 and $100 \mathrm{nM}$, respectively.

\section{Cell viability assay}

LNCaP cells were seeded into 96-well plates at $1 \times 10^{3}$ cells per well and incubated overnight at $37^{\circ} \mathrm{C}$. Cells were transfected with either miR-29b-3p mimics or inhibitors. After $24 \mathrm{~h}$, cells were irradiated with X-rays at dose of $0,1,2,4,6$ or 8 Gy at room temperature. $24 \mathrm{~h}$ after IR, cells were incubated with 5 $\mu \mathrm{g} / \mu \mathrm{L}$ MTT (Sigma-Aldrich) at $37{ }^{\circ} \mathrm{C}$ for $4 \mathrm{~h}$, following which the supernatant was removed and $150 \mu \mathrm{L}$ DMSO (Sigma-Aldrich) was added into each well to dissolve the formazan crystals. The absorbance value was recorded at $490 \mathrm{~nm}$ using a microplate reader (Tecan Infinite M200; Tecan Group, Ltd.).

In addition, cell viability of LNCaP following miRNA mimics or inhibitor transfection and 4Gy $X$-rays irradiation was also measured using the Cell Counting Kit-8 (CCK-8; Dojindo Molecular Technologies, Inc.) at 24,48 and $72 \mathrm{~h}$ after IR at $37^{\circ} \mathrm{C}$, as described previously [18].

\section{Clone survival assay}

As described above, the irradiated cells were seeded in $60 \mathrm{~mm}$ dishes with $2 \times 10^{3}$ cells. Cells were incubated for 13 days and colonies were fixed using $100 \%$ methanol and stained with $0.5 \%$ crystal violet (Sigma-Aldrich) for $30 \mathrm{~min}$ at room temperature. Only colonies consisting of $\geq 50$ cells were counted for each treatment group.

\section{Luciferase assay}

To construct WISP1 3'-untranslated region (UTR)-luciferase plasmid, the 425bp WISP1 3'-UTR fragments containing the miR-29b-binding (WISP1-3'UTR-WT) or WISP1 3'-UTR-mutated (WISP1-3'-UTRMut) site of miR-29b (Table S4) were inserted into the multiple cloning site of pGL3-basic vector, which contains a luciferase reporter gene. DNA sequencing assay identified the sequence (BGI-Tech., Wuhan). $293 \mathrm{~T}$ cells were seeded into 48 -well plates with $50 \%$ confluence $200 \mathrm{ng} / \mu \mathrm{L}$ constructed luciferase vectors and $50 \mathrm{nM}$ miR-29b-3p mimics or negative control (miR-con) were cotransfected into 293T cells using
Lipofectamine 2000 Transfection reagent (Invitrogen, Carlsbad, CA). The cells were lysed $48 \mathrm{~h}$ after transfection. Firefly and Renilla luciferase activities were measured using the Dual-Luciferase ${ }^{\circledR}$ Reporter Assay Kit (Promega Coporation) on the microplate reader according to manufacturer's protocol (Tecan Infinite M200; Tecan Group, Ltd.). Renilla luciferase was used to normalize the firefly luciferase enzyme activity.

\section{Apoptosis assays}

LNCaP cells with $30 \%$ confluence were transfected with either miR-29b-3p mimics or inhibitors for $24 \mathrm{~h}$ and exposed to $4 \mathrm{~Gy}$ X-ray. $1 \times 10^{6}$ cells were harvested $24 \mathrm{~h}$ after IR and stained with FITC-Annexin $\mathrm{V}$ and propidium iodide (PI) at final cell density of $1 \times 10^{3}$ cells $/ \mu \mathrm{L}$ to measure the number of apoptotic/necrotic cells using Annexin V/PI staining kit according to manufacturer's protocol (BD Biosciences). Apoptosis cells were measured by BD FACSVerse ${ }^{\mathrm{TM}}$ and analyzed using the BD FlowJo ${ }^{\circledR}$ software (BD Biosciences).

\section{Western blot}

The cells were lysed using RIPA buffer supplemented with 1\% PMSF (Beyotime Bio.) on ice. Bicinchoninic acid protein assay was performed to determine protein concentration (Pierce; Thermo Fisher Scientific, Inc). $20 \mu \mathrm{g}$ total protein was fractionated and loaded onto 10\% SDS-PAGE and transferred to $0.45 \mu \mathrm{m}$ PVDF membrane. The membranes were blocked with 5\% not-fat milk in buffer for $2 \mathrm{~h}$ at room temperature, and followed by incubation with primary antibodies: WISP1 (ab155654; 1:1,000; Abcam), Bcl-XL (ab32370; 1:5,000; Abcam), cleaved-caspase 3 (9661; 1:1,000; Cell Signaling Technology, Inc.), caspase 3 (9662; 1:1,000; Cell Signaling Technology, Inc.), PARP (9532; 1:1,000; Cell Signaling Technology, Inc.), cleaved PARP (5625; 1:1,000; Cell Signaling Technology, Inc.) and $\beta$-actin (AP0060; 1:5,000; Bioworld Tech., Inc.) overnight at 4 ${ }^{\circ} \mathrm{C}$. Horseradish peroxidase-conjugated anti-rabbit IgG secondary antibody was incubated with membranes for $2 \mathrm{~h}$ at room temperature. The protein blots were visualized using a chemiluminescence kit (EMD Millipore) and photographed using AI680 (Alpha Innotech Corporation). The relative expression of proteins was quantified using Image Quant TL software (version 8.1; GE Healthcare Life Sciences).

\section{Statistical analysis}

Statistical analysis was performed using twotailed Student's $t$-test and two-way ANOVA. Comparing statistical significance of $>2$ groups was analyzed using Tukey's test. Data are presented as 
means $\pm S D(n=3) . P<0.05$ was considered significant difference.

\section{Results}

\section{miR-29b-3p expression is increased in response to IR in LNCaP cells}

To measure the expression alteration of miRNAs in response to IR, LNCaP cells were exposed to 0.5 or 4 Gy X-rays or carbon ions. The expression of miR-29b-3p was upregulated in LNCaP cells following IR (Fig. 1A). To verify this finding, RT-qPCR was performed and showed that miR-29b-3p expression reached its maximum at $24 \mathrm{~h}$ with 4 Gy X-ray irradiation (Fig. $1 \mathrm{~B}$ and C). These results indicated that miR-29b-3p was upregulated and could be involved in response to IR in LNCaP cells.

\section{miR-29b-3p knockdown attenuates the response of LNCaP cells to IR}

To investigate the radiobiological role of miR-29b-3p in LNCaP cells, miR-29b-3p was knocked down by transfecting anti-miR-29b-3p into LNCaP cells. $48 \mathrm{~h}$ after transfection, the expression of miR-29b-3p was significantly reduced (Fig. 2A). After knockdown of miR-29b-3p and X-rays irradiation, cell viability was significantly higher than that in the negative control group (anti-con) (Fig. 2B), whilst IR-induced growth inhibition was attenuated (Fig. 2C). Clone survival of LNCaP cells was increased (Fig. 2D and Figure S1). The results suggested that knockdown of miR-29b-3p reduced the cellular response to IR, leading to radioresistance.

\section{Overexpression of $\mathrm{miR}-29 \mathrm{~b}-3 \mathrm{p}$ enhances the radiosensitivity of $\mathrm{LNCaP}$ cells}

To verify the role of miR-29b-3p in response to IR, miR-29b-3p mimics were transfected into LNCaP cells to overexpress it. As shown in Fig.3A, miR$29 b-3 p$ mimics markedly increased the expression of miR-29b-3p. Overexpression of miR-29b-3p reduced cell viability (Fig. 3B), suppressed proliferation (Fig. 3C) and reduced clone survival (Fig. 3D and Figure S2) in LNCaP cells after exposure to X-rays. These findings demonstrated that overexpression of miR-29b-3p enhanced radiosensitivity of LNCaP cells.

\section{miR-29b-3p suppresses WISPI expression by directly targeting the 3'-UTR of WISPI mRNA}

miRNAs function to regulate gene expression through binding to mRNA targets. In terms of miR-29b-3p, target prediction using three databases (TargetScan, miRanda, RNA22) revealed a perfect match between the seed region of miR-29b-3p and the 3'-UTR of WISP1 mRNA (Fig.4A). To conform the
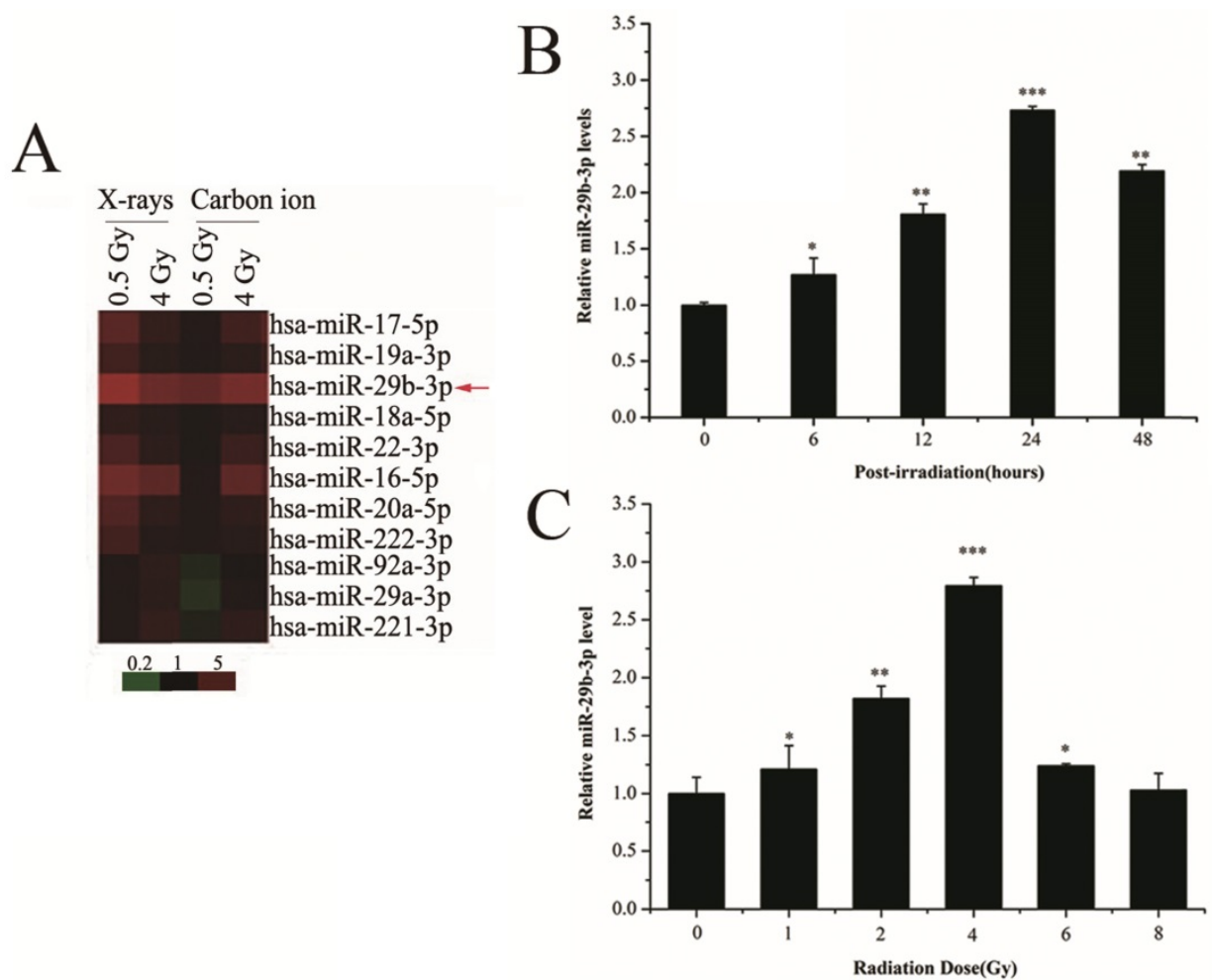

Figure 1. miR-29b-3p expression is increased in response to IR in LNCaP cells. (A) A mini heat map of the miRNA expression profile in LNCaP cells exposed to either 0.5 or 4 Gy X-rays or carbon ion beam irradiation. Red arrow indicates the location of miR-29b-3p, the expression of which is upregulated in response to IR. (B) The levels of miR-29b-3p expression in LNCaP cells at indicated time points following exposure to $4 \mathrm{~Gy}$ X-ray irradiation. (C) Levels of miR-29b-3p expression in LNCaP cells $24 \mathrm{~h}$ after irradiation with indicated doses of $\mathrm{X}$-rays. $* P<0.05, * * P<0.01$ and $* * * P<0.001$ vs. $0 \mathrm{~h}$ or $0 \mathrm{~Gy}$. 
prediction, pGL3-WISP1-3'-UTR-WT and pGL3WISP1-3'-UTR-Mut vectors were constructed. As shown in Fig. 4B, miR-29b-3p mimics significantly reduced the luciferase activity in 293T cells cotransfected with pGL3-WISP1-3'-UTR-WT, but did not affect that in cells cotransfected with the pGL3WISP1-3'-UTR-Mut vector. The result supported the prediction that miR-29b-3p targeted WISP1 through directly binding to its $3^{\prime}-\mathrm{UTR}$. To further verify the direct relationship between WISP1 and miR-29b-3p, WISP1 mRNA and protein expression were measured after transfecting of miR-29b-3p mimics. Compared with miR-con group, the expression levels of both WISP1 mRNA and protein were significantly reduced in cells transfected with the miR-29b-3p mimics (Fig. $4 \mathrm{C}$ and D), validating miR-29b-3p targeting downregulated WISP1 expression.

\section{miR-29b-3p overexpression enhances IR-induced apoptosis by modulating the WISP1-mediated mitochondrial apoptosis pathway}

Overexpression of WISP1 could accelerate cell growth and inhibit apoptosis by suppressing the activation of caspase 3 and release of cytochrome C [29]. Therefore, the effect of miR-29b-3p on IR-induced apoptosis may be associated with
WISP1-mediated mitochondrial apoptosis pathway. Indeed, as shown in Fig. 5A and B, overexpression of miR-29b-3p dramatically increased IR-induced apoptosis in LNCaP cells. Interestingly, miR-29b-3p itself could also increase apoptosis in the absence of IR when compared with cells transfected with miR-con. While knockdown of miR-29b-3p had the opposite effect, that is to say, anti-miR-29b-3p attenuated IRcaused apoptosis.

To unravel the possible molecular mechanism underlying the miR-29b-3p-mediated enhancement in IR-induced apoptosis, expression of a number of protein components related with mitochondrial apoptosis pathway was measured. Overexpression of miR-29b-3p markedly reduced expression of WISP1 and Bcl-XL, but increased the activation of caspase 3 and PARP. X-ray irradiation further reduced expression of WISP1 and Bcl-XL, whereas activated caspase 3 and PARP (Fig. 5D). By contrast, transfecting miR-29b-3p inhibitors into LNCaP cells resulted in the opposite effects (Fig. 5C). The results suggested that miR-29b-3p enhanced IR-induced apoptosis by suppressing WISP1 expression, activating mitochondrial apoptosis pathway through modulating the Bcl-XL expression and activation of caspase 3 and PARP.
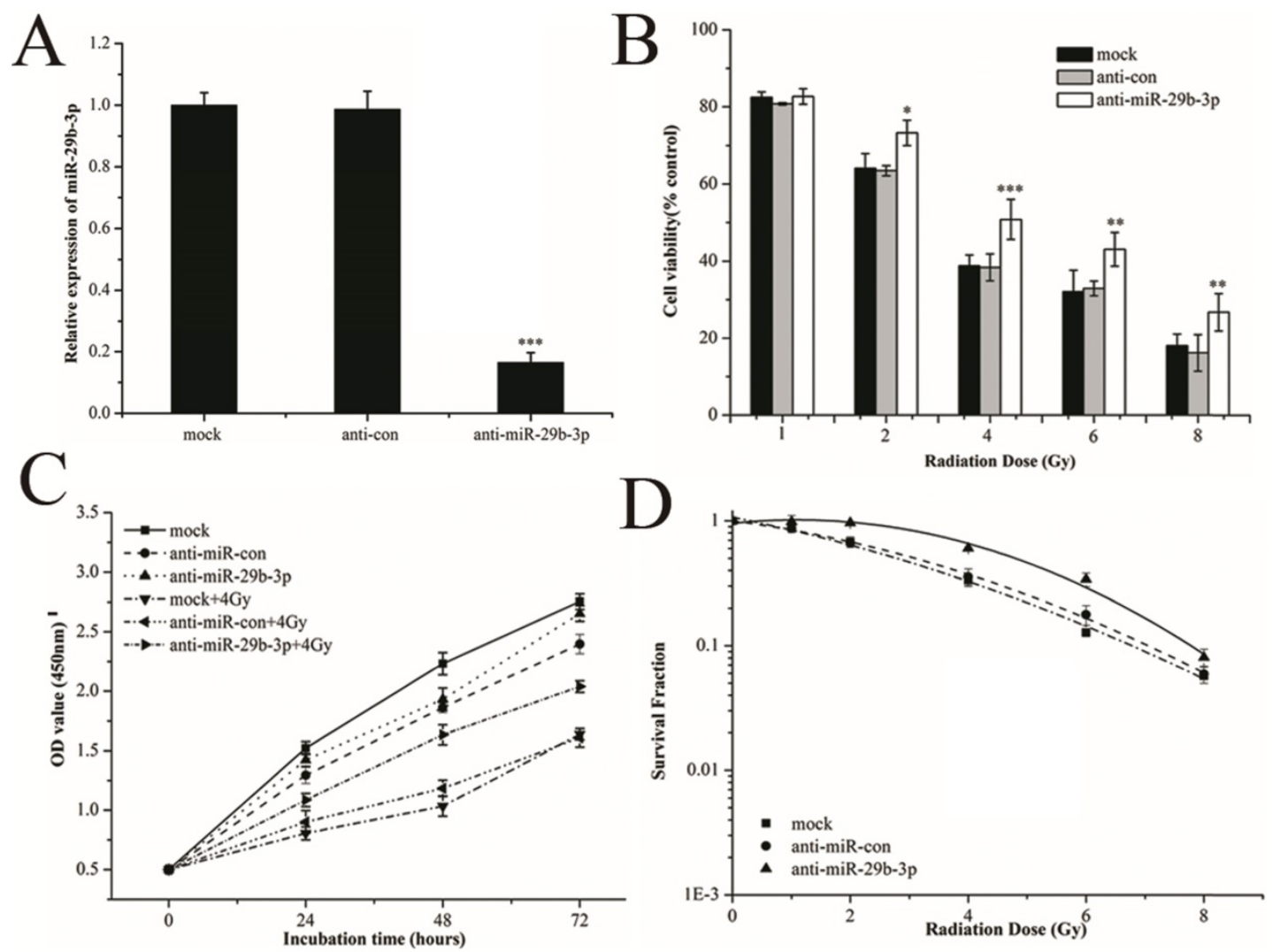

Figure 2. miR-29b-3p knockdown attenuates response of LNCaP cells to IR. (A) The relative levels of miR-29b-3p expression $48 \mathrm{~h}$ after transfection with miR-29b-3p inhibitors or negative control. (B) Cell viability, (C) Cell proliferation and (D) clonogenic ability in LNCaP cells were measured following transfection with anti-miR-29b-3p and X-ray exposure. $* P<0.05, * * P<0.01$ and $* * * P<0.001$ vs. negative control group (anti-con). 

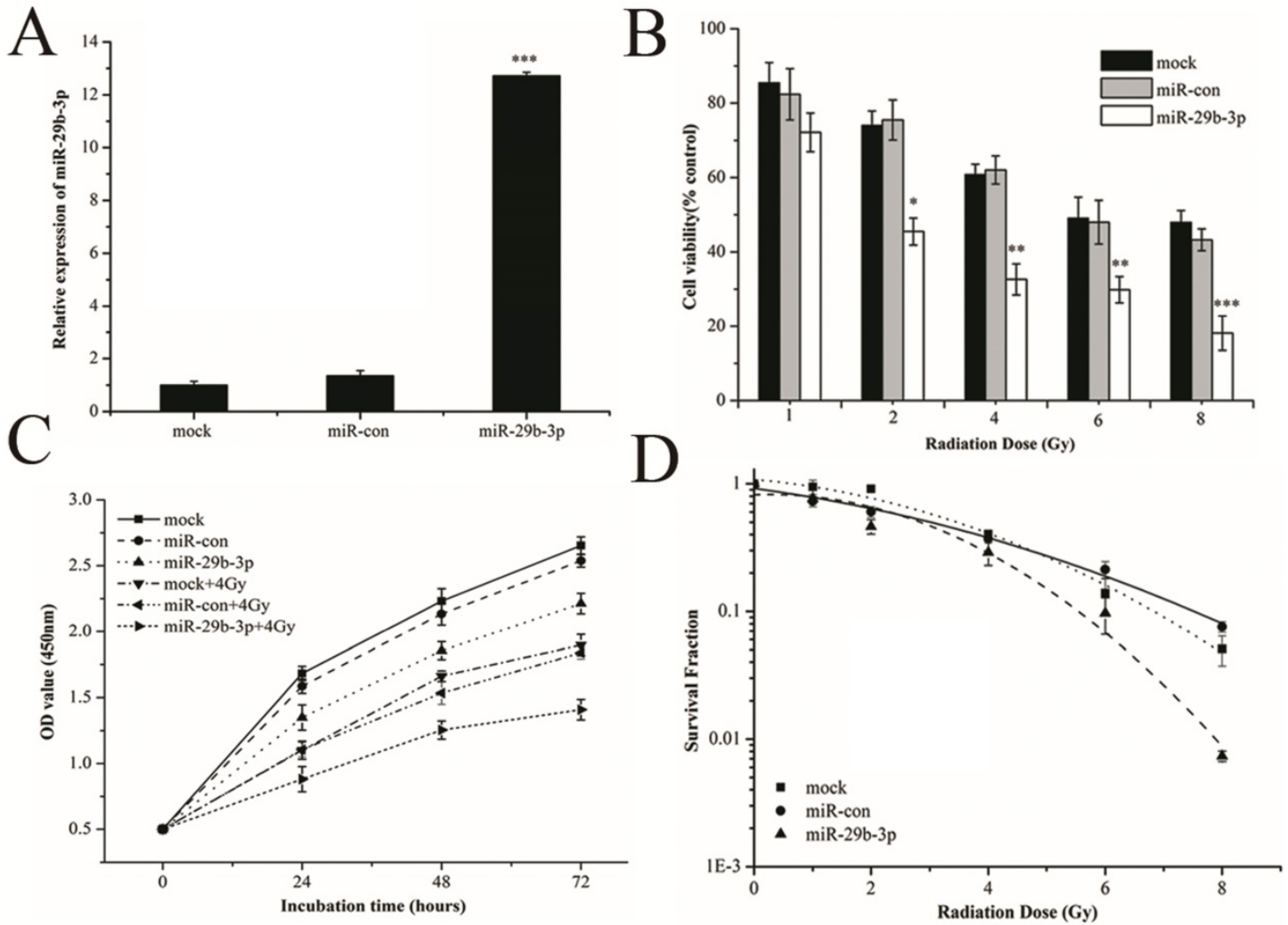

Figure 3. Overexpression of miR-29b-3p enhances radiosensitivity in LNCaP cells. (A) Relative levels of miR-29b-3p expression $48 \mathrm{~h}$ following transfection with the miR-29b-3p mimic or negative control. (B) Cell viability, (C) Cell proliferation and (D) Clonogenic survival was measured in LNCaP cells overexpressing miR-29b-3p and treatment with indicated doses of $X$-ray irradiation. $* P<0.05, * * P<0.01$ and $* * * P<0.001$ vs. negative control group (miR-con).
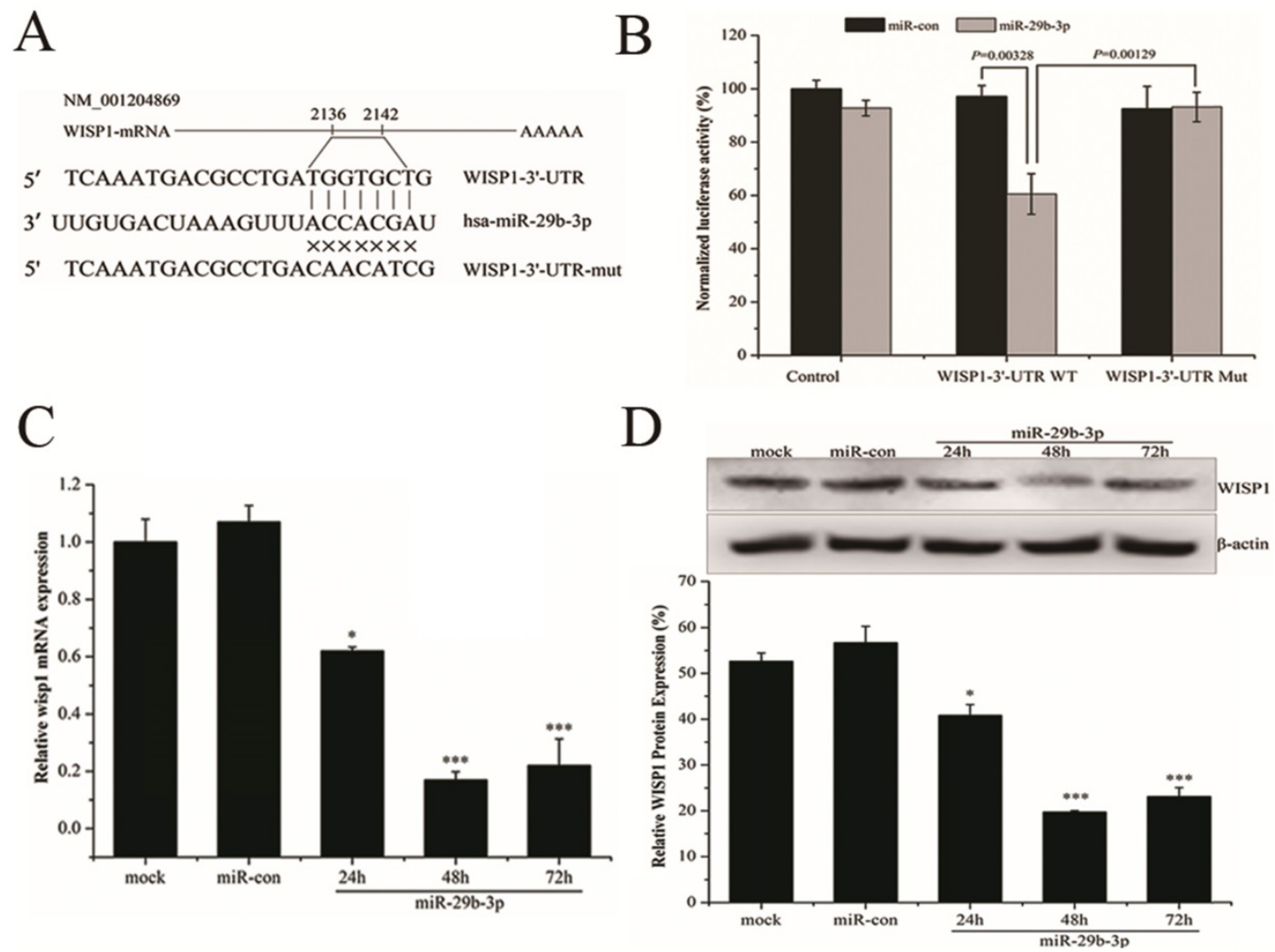

Figure 4. miR-29b-3p suppresses WISPI expression by targeting 3'-UTR of WISPI. (A) Putative miR-29b-3p binding site in the 3'-UTR of human WISPI mRNA. Sequence of the mature miR-29b-3p was aligned against the corresponding target site on the WT and the MUT 3'-UTR of the WISPI mRNA. (B) Luciferase activity was measured $48 \mathrm{~h}$ after cotransfection with the pGL3 vector encoding WT or MUT WISP1 3'-UTR and miR-con or miR-29b-3p mimics. Relative expression of WISP1 (C) mRNA and (D) protein in LNCaP cells following transfection with miR-29b-3p mimics or miR-con. $* P<0.05$ and $* * * P<0.001$ vs. negative control group (miR-con). 

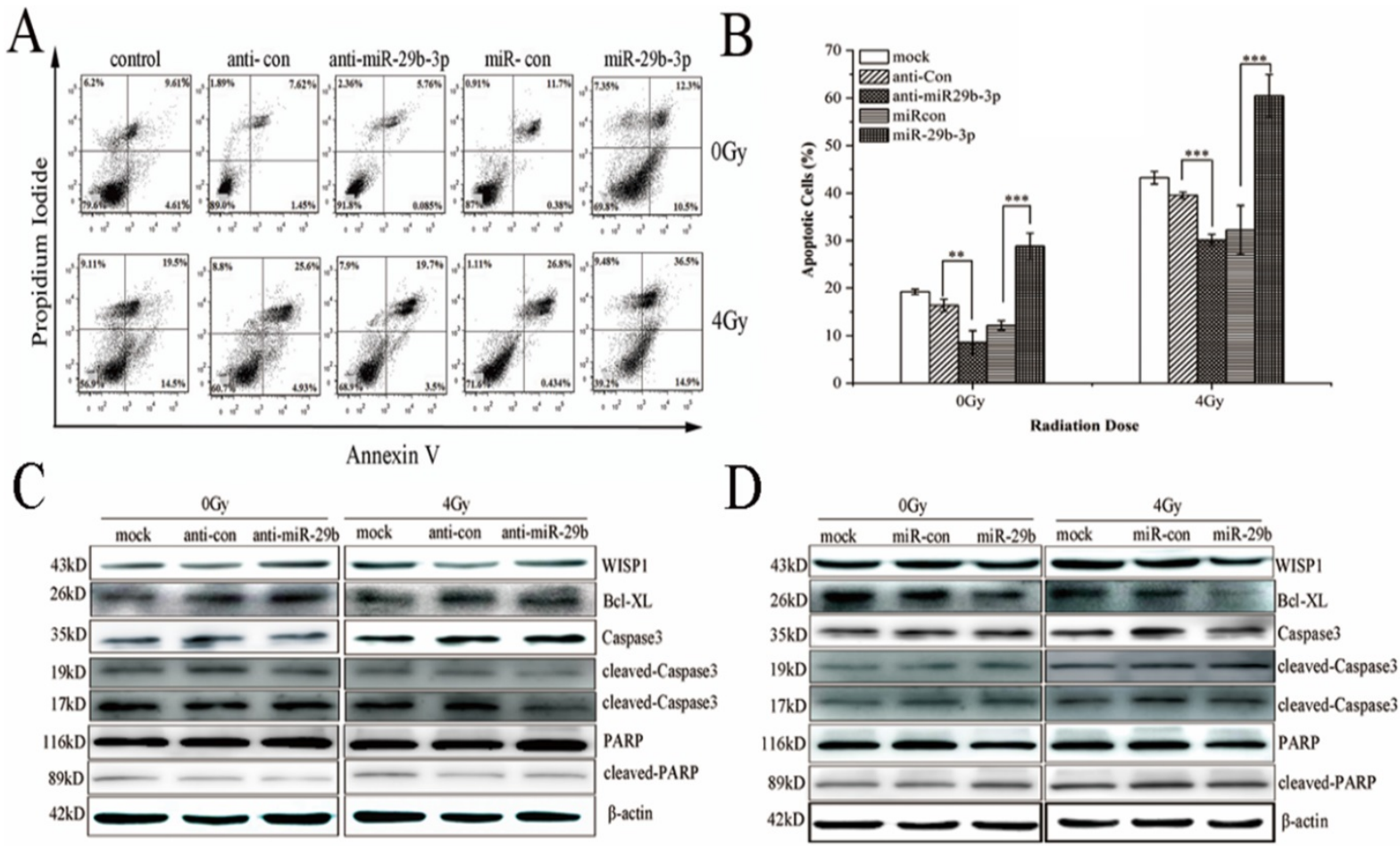

Figure 5. miR-29b-3p enhances IR-induced apoptosis by suppressing WISPI expression and activating the mitochondrial apoptosis pathway. (A) Representative flow cytometry dot plots showing apoptotic LNCaP cells transfeced with miR-29b-3p inhibitors or mimics 24h before 4 Gy X-rays irradiation. (B) Quantification of (A). (C) miR-29b-3p knockdown increased the expression levels of WISPI and Bcl-XL whilst blocking the activation of caspase 3 and PARP in response to IR. (D) miR-29b-3p overexpression reduced WISPI and $\mathrm{Bcl}-\mathrm{XL}$ expression, whilst inducing the activation of caspase3 and PARP in response to IR. $* * P<0.01$ and $* * * P<0.001$ vs. negative control group.

\section{Discussion}

Accumulating evidence has demonstrated that the expression level of miR-29b is lower in variety of malignancies and cancer cell lines than that in normal tissues or cell lines [21]. miR-29b expression has been correlated with clinicopathological characteristics in patients with colorectal cancer [30-32], breast cancer [33], and osteosarcoma [34] patients, suggesting its potential to serve as a biomarker for diagnosis and prognosis of cancer. miR-29b has been documented to negatively affect tumorigenesis and cancer progression by regulating cell proliferation, survival, and metastasis [28], Whilst overexpression of miR-29b can also inhibit tumorigenesis [35] and angiogenesis [36-38]. Moreover, miR-29b can reverse drug resistance in ovarian cancer $[39,40]$ and colorectal cancer [41], as well as radioresistance in cervical cancer [42]. In this study, we found that overexpression of miR-29b-3p reduced cell viability, suppressed cell proliferation, and decreased colony formation, resulting in increased sensitivity of LNCaP cells to X-rays irradiation. Based on the experimental and clinical evidence, miR-29b can be potentially explored as a targeted therapeutic agent.

Prostate cancer is the most common cancer among man, where radiotherapy is currently the only definitive treatment option for patients with localized prostate cancer [2]. However, resistance to radio- therapy remains a major obstacle that limits the efficacy of this treatment. To improve the outcome of radiotherapy and enhance radiosensitivity of prostate cancer cells, understanding the mechanisms underlying cellular radioresistance is currently a hot topic of research. In the present study, miR-29b-3p expression was found to be significantly increased in response to X-rays and carbon ions irradiation in LNCaP cells, suggesting that miR-29b-3p participate in the response to IR. Gain- and loss-of-function experiments next verified that overexpression of miR-29b-3p reduced cell viability, suppressed cell proliferation, and decreased colony formation, resulting in increased sensitivity of LNCaP cells to $X$-rays irradiation. By contrast, knockdown of miR-29b-3p expression resulted in the opposite effects. To the best of our knowledge, this is the first work showing miR-29b-3p upregulated in response to IR and overexpression of miR-29b-3p enhanced the radiosensitivity of LNCaP cells. Unfortunately, in this study, the function of miR-29b-3p in response to only $\mathrm{X}$-ray irradiation was investigated due to restrictions on the use of the carbon ion beam radiation source at the HIRFL. The mechanistic function of miR-29b-3p in mediating the response to carbon ion radiation requires further study.

WISP-1, as a member of the CCN family of growth factors, plays a critical role in tumorigenesis and development of various cancers $[3,43]$. WISP1 has 
been demonstrated to involve in cell proliferation, apoptosis, invasion and metastasis [44]. Supporting this, a number of studies revealed that WISP1 was associated with the formation and evolution of lung cancer [45], hepatocellular carcinoma [46] and colorectal cancer [4]. The ectopic expression of WISP1 has also been observed in a number of cancers, including prostate cancer [7]. Inhibition of WISP1 function using neutralizing antibodies reduced prostate cancer cell growth and inhibited metastasis to bone [7]. Consistent with these findings, osteoblastderived WISP1 has also been shown to promote migration and enhance cell adhesion to the bone through VCAM-1/a4 $\beta 1$ integrin pathway in prostate cancer cells $[8,9]$. Therefore, WISP1 can serve as a potential therapeutic target to prostate cancer patients. Interestingly, WISP1 was found to contribute fractionated IR-induced radioresistance in xenograft tumor models, where targeting WISP1 using antibody or siRNA sensitized esophageal carcinoma cells to IR [10-12]. In addition, IR-induced aberrant activation of the Wnt/ $\beta$-catenin pathway was associated with radioresistance $[13,14]$. In our study, WISP1 was predicted and subsequently verified to be a direct target of miR-29b-3p. MiR-29b-3p enhanced sensitivity of LNCaP cells to X-rays irradiation by directly inhibiting WISP1 expression.

Previous study has suggested WISP1 attenuated p53-mediated apoptosis by upregulating the expression of anti-apoptotic Bcl-XL but blocking activation of caspase 3 [29]. This study showed that inhibition of WISP1 by miR-29b-3p overexpression markedly reduced Bcl-XL expression but significantly activated caspase 3 and PARP in LNCaP cells following $4 \mathrm{~Gy}$ X-rays irradiation. It was speculated that miR-29b-3p enhanced IR-induced apoptosis by suppressing WISP1 expression, leading to activation of the mitochondrial apoptosis pathway.

\section{Conclusion}

In summary, miR-29b-3p was significantly upregulated in response to IR. Overexpression of miR-29b-3p enhanced sensitivity of LNCaP cells to $\mathrm{X}$-rays irradiation. WISP1 was identified as a direct target of miR-29b-3p. miR-29b-3p enhanced the radiosensitivity through modulating WISP1-mediated mitochondrial apoptosis (showing reduced Bcl-XL and activation of caspase 3 and PARP) (Fig. 6). The results revealed a novel mechanism of miR-29b-3p enhanced radiosensitivity by target-regulating WISP1 expression in response to IR. These findings may provide an alternative treatment to overcome radioresistance for prostate cancer patients, in particular for those with higher level of WISP1 expression.

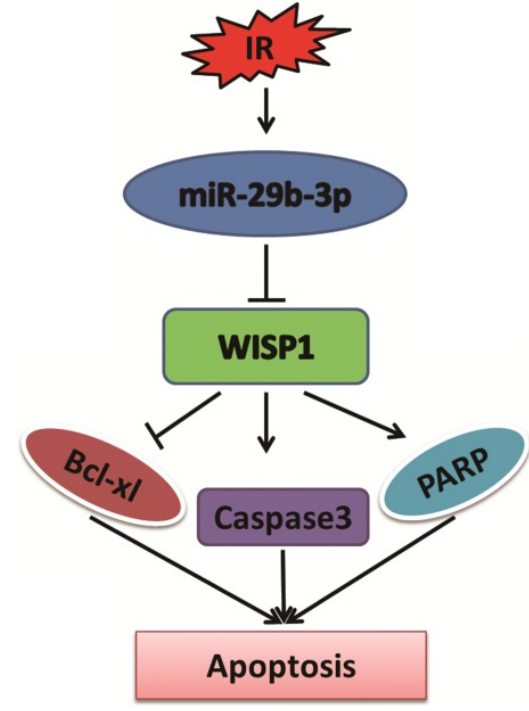

Figure 6. The function of miR-29b-3p and WISPI in IR-induced apoptosis. miR-29b-3p expression is upregulated in $\mathrm{LNCaP}$ cells in response to IR. Elevated miR-29b-3p suppresses WISPI expression by directly targeting the 3'-UTR of WISPI mRNA, which then activates the mitochondrial apoptosis pathway by suppressing $\mathrm{Bcl}-\mathrm{XL}$ expression, but activating Caspase 3 and PARP, resulting in increased LNCaP cell apoptosis.

\section{Supplementary Material}

Supplementary figures and tables. http://www.jcancer.org/v11p6356s1.pdf

\section{Acknowledgments}

This work was supported by grants from National Key R\&D project of China (2018YFE0205100), the National Natural Science Foundation of China (11665003), and Cancer Research Youth Science Foundation of Chinese Anti-Cancer Association (CAYC18A06).

\section{Authors' contributions}

$\mathrm{HZ}$ and AM conceived and designed this study. JT, FW, DT and HY performed the experiments. CT, CS and JS analyzed the data. SL and XX performed data analysis and interpretation. AM and JT wrote the paper.

\section{Competing Interests}

The authors have declared that no competing interest exists.

\section{References}

1. Siegel RL, Miller KD, Jemal A. Cancer statistics, 2020. CA Cancer J Clin. 2020; 70: 7-30.

2. Palacios DA, Miyake M, Rosser CJ. Radiosensitization in prostate cancer: mechanisms and targets. BMC Urol. 2013; 13: 4.

3. $\mathrm{Xu} \mathrm{L}$, Corcoran RB, Welsh JW, et al. WISP-1 is a Wnt-1- and beta-catenin-responsive oncogene. Genes \& development. 2000; 14: 585-95.

4. Wu J, Long Z, Cai H, et al. High expression of WISP1 in colon cancer is associated with apoptosis, invasion and poor prognosis. Oncotarget. 2016; 7: 49834-47.

5. Jing $\mathrm{D}$, Zhang $\mathrm{Q}, \mathrm{Yu} \mathrm{H}$, et al. Identification of WISP1 as a novel oncogene in glioblastoma. Int J Oncol. 2017; 51: 1261-70. 
6. Jung EK, Kim SA, Yoon TM, et al. WNT1-inducible signaling pathway protein-1 contributes to tumor progression and treatment failure in oral squamous cell carcinoma. Oncol Lett. 2017; 14: 1719-24.

7. Ono M, Inkson CA, Sonn R, et al. WISP1/CCN4: a potential target for inhibiting prostate cancer growth and spread to bone. PLoS One. 2013; 8: e71709.

8. Tai HC, Chang AC, Yu HJ, et al. Osteoblast-derived WNT-induced secreted protein 1 increases VCAM-1 expression and enhances prostate cancer metastasis by down-regulating miR-126. Oncotarget. 2014; 5: 7589-98.

9. Chang AC, Chen PC, Lin YF, et al. Osteoblast-secreted WISP-1 promotes adherence of prostate cancer cells to bone via the VCAM-1/integrin alpha4beta1 system. Cancer letters. 2018; 426: 47-56.

10. Zhang $\mathrm{H}$, Luo $\mathrm{H}$, Jiang $\mathrm{Z}$, et al. Fractionated irradiation-induced EMT-like phenotype conferred radioresistance in esophageal squamous cell carcinoma. J Radiat Res. 2016; 57: 370-80.

11. Zhang $\mathrm{H}$, Luo $\mathrm{H}, \mathrm{Hu} \mathrm{Z}$, et al. Targeting WISP1 to sensitize esophageal squamous cell carcinoma to irradiation. Oncotarget. 2015; 6: 6218-34.

12. $\mathrm{Li} \mathrm{WF}$, Zhang $\mathrm{L}, \mathrm{Li} \mathrm{HY}$, et al. WISP-1 contributes to fractionated irradiation-induced radioresistance in esophageal carcinoma cell lines and mice. PLoS One. 2014; 9: e94751.

13. Kim Y, Kim KH, Lee J, et al. Wnt activation is implicated in glioblastoma radioresistance. Laboratory investigation; a journal of technical methods and pathology. 2012; 92: 466-73

14. Chen MS, Woodward WA, Behbod F, et al. Wnt/beta-catenin mediates radiation resistance of $\mathrm{Sca} 1^{+}$progenitors in an immortalized mammary gland cell line. Journal of cell science. 2007; 120: 468-77.

15. Ohtsuka M, Ling H, Doki Y, et al. MicroRNA Processing and Human Cancer. Journal of clinical medicine. 2015; 4: 1651-67.

16. Di Leva G, Croce CM. miRNA profiling of cancer. Current opinion in genetics \& development. 2013; 23: 3-11.

17. Mao A, Liu Y, Wang $\mathrm{Y}$, et al. miR-449a enhances radiosensitivity through modulating pRb/E2F1 in prostate cancer cells. Tumour biology : the journal of the International Society for Oncodevelopmental Biology and Medicine. 2016; 37: 4831-40.

18. Mao A, Zhao Q, Zhou X, et al. MicroRNA-449a enhances radiosensitivity by downregulation of c-Myc in prostate cancer cells. Scientific reports. 2016; 6: 27346.

19. Wang F, Mao A, Tang J, et al microRNA-16-5p enhances radiosensitivity through modulating Cyclin D1/E1-pRb-E2F1 pathway in prostate cancer cells. J Cell Physiol. 2019; 234: 13182-90.

20. Kriegel A, Liu Y, Fang Y, et al. The miR-29 family: genomics, cell biology, and relevance to renal and cardiovascular injury. Physiological genomics. 2012; 44: $237-44$.

21. Jiang H, Zhang G, Wu JH, et al. Diverse roles of miR-29 in cancer (review). Oncology reports. 2014; 31: 1509-16.

22. Sur S, Steele R, Shi X, et al. miRNA-29b Inhibits Prostate Tumor Growth and Induces Apoptosis by Increasing Bim Expression. Cells. 2019; 8.

23. Zhu C, Hou X, Zhu J, et al. Expression of miR-30c and miR-29b in prostate cancer and its diagnostic significance. Oncol Lett. 2018; 16: 3140-4.

24. Steele R, Mott JL, Ray RB. MBP-1 upregulates miR-29b that represses Mcl-1, collagens, and matrix-metalloproteinase- 2 in prostate cancer cells. Genes \& cancer. 2010; 1 : 381-7.

25. Ivanovic RF, Viana NI, Morais DR, et al. miR-29b enhances prostate cancer cell invasion independently of MMP-2 expression. Cancer cell international. 2018; 18: 18.

26. Yan B, Guo Q, Nan XX, et al. Micro-ribonucleic acid $29 \mathrm{~b}$ inhibits cell proliferation and invasion and enhances cell apoptosis and chemotherapy effects of cisplatin via targeting of DNMT3b and AKT3 in prostate cancer. OncoTargets and therapy. 2015; 8: 557-65.

27. Ru P, Steele R, Newhall P, et al. miRNA-29b suppresses prostate cancer metastasis by regulating epithelial-mesenchymal transition signaling. Molecular cancer therapeutics. 2012; 11: 1166-73.

28. Yan B, Guo Q, Fu FJ, et al. The role of miR-29b in cancer: regulation, function, and signaling. OncoTargets and therapy. 2015; 8: 539-48.

29. Su F, Overholtzer M, Besser D, et al. WISP-1 attenuates p53-mediated apoptosis in response to DNA damage through activation of the Akt kinase. Genes \& development. 2002; 16: 46-57.

30. Inoue A, Yamamoto $\mathrm{H}$, Uemura $\mathrm{M}$, et al. MicroRNA-29b is a Novel Prognostic Marker in Colorectal Cancer. Annals of surgical oncology. 2015; 22 Suppl 3: S1410-8.

31. Li L, Guo Y, Chen Y, et al. The Diagnostic Efficacy and Biological Effects of microRNA-29b for Colon Cancer. Technology in cancer research \& treatment. 2016; 15: 772-9.

32. Basati G, Razavi AE, Pakzad I, et al. Circulating levels of the miRNAs, miR-194, and miR-29b, as clinically useful biomarkers for colorectal cancer. Tumour biology : the journal of the International Society for Oncodevelopmental Biology and Medicine. 2016; 37: 1781-8.

33. Papachristopoulou G, Papadopoulos EI, Nonni A, et al. Expression Analysis of miR-29b in Malignant and Benign Breast Tumors: A Promising Prognostic Biomarker for Invasive Ductal Carcinoma With a Possible Histotype-Related Expression Status. Clinical breast cancer. 2018; 18: 305-12.e3.

34. Hong $\mathrm{Q}$, Fang J, Pang $\mathrm{Y}$, et al. Prognostic value of the microRNA-29 family in patients with primary osteosarcomas. Medical oncology (Northwood, London, England). 2014; 31: 37
35. Shin J, Shim HG, Hwang $\mathrm{T}$, et al. Restoration of miR-29b exerts anti-cancer effects on glioblastoma. Cancer cell international. 2017; 17: 104

36. Li Y, Cai B, Shen L, et al. MiRNA-29b suppresses tumor growth through simultaneously inhibiting angiogenesis and tumorigenesis by targeting Akt3. Cancer letters. 2017; 397: 111-9.

37. Wang H, Guan X, Tu Y, et al. MicroRNA-29b attenuates non-small cell lung cancer metastasis by targeting matrix metalloproteinase 2 and PTEN. Journal of experimental \& clinical cancer research : CR. 2015; 34: 59.

38. Li Y, Zhang Z, Xiao Z, et al. Chemotherapy-mediated miR-29b expression inhibits the invasion and angiogenesis of cervical cancer. Oncotarget. 2017; 8: 14655-65.

39. Dai F, Zhang Y, Zhu X, et al. The anti-chemoresistant effect and mechanism of MUC1 aptamer-miR-29b chimera in ovarian cancer. Gynecologic oncology. 2013; 131: 451-9.

40. Sugio A, Iwasaki M, Habata S, et al. BAG3 upregulates Mcl-1 through downregulation of miR-29b to induce anticancer drug resistance in ovarian cancer. Gynecologic oncology. 2014; 134: 615-23.

41. Liu H, Cheng XH. MiR-29b reverses oxaliplatin-resistance in colorectal cancer by targeting SIRT1. Oncotarget. 2018; 9: 12304-15.

42. Zhang $T$, Xue $X$, Peng $H$. Therapeutic Delivery of miR-29b Enhances Radiosensitivity in Cervical Cancer. Molecular therapy : the journal of the American Society of Gene Therapy. 2019; 27: 1183-94

43. Berschneider B, Konigshoff M. WNT1 inducible signaling pathway protein 1 (WISP1): a novel mediator linking development and disease. The international journal of biochemistry \& cell biology. 2011; 43: 306-9.

44. Liu Y, Song Y, Ye M, et al. The emerging role of WISP proteins in tumorigenesis and cancer therapy. Journal of translational medicine. 2019; 17:

45. Chen PP, Li WJ, Wang Y, et al. Expression of Cyr61, CTGF, and WISP-1 correlates with clinical features of lung cancer. PLoS One. 2007; 2: e534.

46. Zhang $\mathrm{H}, \mathrm{Li} \mathrm{W}$, Huang $\mathrm{P}$, et al. Expression of $\mathrm{CCN}$ family members correlates with the clinical features of hepatocellular carcinoma. Oncology reports. 2015; 33: $1481-92$ 